\title{
Bringing genetics into primary care: findings from a national evaluation of pilots in England
}

\author{
Graham P. Martin, Graeme Currie \&o Rachael Finn
}

Objectives: Developments in genetic knowledge and clinical applications are seen as rendering traditional modes of organising genetics provision increasingly inappropriate. In common with a number of developed world countries the United Kingdom has sought to increase the role of primary care in delivering such services. However, efforts to reconfigure service delivery face multiple challenges associated with divergent policy objectives, organisational boundaries, and professional cultures. This paper presents findings from an evaluation of an English initiative to integrate genetics into 'mainstream' clinical provision in the National Health Service.

Methods: Qualitative research in 11 case-study sites focusing on attempts by pilots funded by the initiative to embed knowledge and provision within primary care illustrating barriers faced and the ways in which these were surmounted.

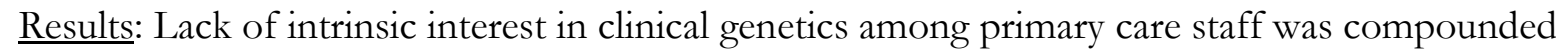
by national targets that focused their attention elsewhere and by service structures that rendered genetics a peripheral concern demanding minimal engagement. Established divisions between the commissioning of mainstream and specialist services, along with the pressures of shorterterm targets, impeded ongoing funding.

Conclusions: More wide-ranging policy and organisational support is required if the aim of entrenching genetics knowledge and practice across the Health Service is to be realised.

$\begin{array}{ll}\text { Published in: } & \text { Journal of Health Services Research \& Policy 14(4): 204-211 } \\ & \begin{array}{l}\text { http:///hshrp.rsmjournals.com// } \\ \text { http:///hrpr.rsmjournals.com/cgi/content/abstract/14/4/204 } \\ \text { doi:10.1258/jhsrp.2009.008158 }\end{array}\end{array}$

\section{Introduction}

The implications of developments in genetics knowledge for human health are demanding the attention of policy makers in the United Kingdom (UK) and elsewhere. The mapping of the Human Genome, among other developments associated with the 'new genetics', has precipitated a rapid increase in knowledge about genetic causation in illness and disability, while the public is increasingly informed about such developments and their potential relevance to health. There are implications too for the organisation of health services. The traditional role of clinical genetics as an esoteric speciality concerned with rare conditions primarily caused by single-gene disorders is being supplemented by a growing awareness of the relevance of genetics to a wide range of common illnesses, so that health service professionals across clinical disciplines and within primary care can draw on knowledge of genetic causation to treat patients appropriately, offer informed advice and refer as necessary. 
In England, concerns of these kinds gave rise in 2003 to a White Paper on future genetics provision, Our Inheritance, Our Future. ${ }^{1}$ The White Paper heralded plans to assist the 'mainstreaming' of genetics knowledge and services throughout the National Health Service (NHS), "assisting genetics to permeate all branches of medicine by supporting new initiatives in genetics based care in key disease areas, in secondary and primary care," and "helping to empower frontline staff and commissioners in the NHS through investment in education and training." "Such developments were seen as a necessary response to the expansion in genetics knowledge and the growth in clinical applications that would follow to divide work appropriately according to skill mix across primary, secondary and tertiary care. "Over time, as the relevance of genetics across the spectrum of other medical specialities becomes apparent, mainstream NHS services will be able to take advantage of new genetic tools in diagnosing, preventing and treating disease." 1 The White Paper announced around $f 8$ million of funding for a programme of pilot services to put into practice these 'mainstreaming' ambitions.

Collaborative working across the sectors, organisations and professional groups of health service bureaucracies, however, is not a straightforward enterprise. Previous research has noted how different work cultures may combine with the divisive effects of structural boundaries to negate efforts to promote collaboration. ${ }^{2,3,4}$ Notable, for example, is how markets and quasimarkets may encourage clinicians to view each other more as competitors than collaborators, whilst driving a wedge between purchasers and providers. Similarly, the centrally-driven performance management of a health care bureaucracy such as the English NHS, deploying targets to drive change in clinicians' and managers' behaviour, may encourage practitioners to focus on core work rather than look outward towards joining their provision with other sectors, specialities or organisations within and beyond the Health Service. ${ }^{4}$ Such difficulties have affected other recent efforts in the UK to promote joined up working between hospitals and primary care (and other agencies, such as social services), for example, in the case of delayed discharges of older people ${ }^{5}$ and chronic disease management. ${ }^{6}$ In relation to genetics, specifically, there are particular barriers to the spread of knowledge and practice to primary care, such as low skills and lack of interest among practitioners, ${ }^{7,8}$ and scepticism about value for patients, ${ }^{9}$ in the UK and elsewhere.

This article presents findings from an evaluation involving qualitative, comparative casestudy research which accompanied the pilot programme following the genetics White Paper. It focuses on the challenges faced by pilots in bridging the divide between community-based services and traditional hospital-based provision, and the ways in which they dealt with these.

The programme of pilots consisted of four streams and 32 pilot sites, and was funded by the English Department of Health $(\mathrm{DH})$. One stream, comprising five sites, involved piloting a method of identifying relatives of those diagnosed with a common inherited disorder, familial hypercholesterolaemia. ${ }^{10}$ A second stream of 10 sites involved service development projects which incorporated genetics into provision in other clinical fields in secondary and primary care. Thirdly, seven pilots, co-funded with Macmillan Cancer Support, implemented a new care pathway for people at possible risk of inherited cancer, across primary, secondary and tertiary care. ${ }^{11}$ Finally, 10 general practitioner (GP) with a special interest (GPSI) in genetics pilots were funded to enable these GPs to provide education and leadership around genetics, alongside clinical services in most cases, within primary care. While most pilots were led by hospital-based staff in secondary or tertiary care, some-notably the GPSI initiatives, but also a few in other streams - were hosted by Primary Care Trusts (PCTs) and led by primary care practitioners.

\section{Methods}

The DH-commissioned evaluation involved the selection of 11 case-study sites from the service development, cancer genetics and GPSI streams for comparative analysis. ${ }^{12}$ These cases were theoretically sampled to ensure variation in characteristics such as clinical field, host organisation 
(PCT, 'mainstream' clinical department in a hospital, or specialist genetics centre) and profession of pilot lead (clinical geneticist, other medical specialist, GP, nurse or manager) (see Table 1). The research involved a combination of qualitative methods, including in-depth interviews with stakeholders (project leads and staff, steering-group members, clinicians and managers in other organisations relating to the projects, commissioners and involved service users), observation at pilot- and programme-level meetings, and analysis of bid documents, meeting notes, local evaluation reports and other relevant documents. Interviews were semi-structured, focusing on questions about organisational set-up, relationships with key stakeholders, leadership, knowledge management, policy drivers, service-user involvement and sustainability, but allowing for deviation from these topics according to the role and knowledge of interviewees. Towards the end of the pilot period, follow-up interviews were conducted with leads in each site, to review progress towards aims and reflect on challenges faced.

Across the cases and including some respondents responsible for the programme as a whole, 125 interviews were conducted. Along with notes from observational work and documentary evidence, these were analysed iteratively, on a case-by-case basis, with at least two authors in each case rereading and coding transcripts, notes and documents, generating themes, and cross-checking these in discussion with each other. The topics addressed in the interview schedule provided a preliminary coding framework and this was supplemented in the course of analysis by other themes identified by the authors. Transcripts and documents were coded accordingly, and excerpts from each source were grouped thematically and analysed, to permit cross-case as well as within-case analysis. As a means of authenticating the analysis, findings were presented to the commissioners of, and some participants in, the research, providing a check on the validity of analysis.

\section{Results}

\section{Engaging primary care in genetics issues}

For pilots based in hospitals and primary care alike, there were some common challenges in gaining the engagement of GPs, nurses and other primary care practitioners in their new services. In general terms, there was notable reluctance among such practitioners to concern themselves with questions of genetics, given other more pressing priorities. Genetics was seen by many primary care practitioners as of little relevance to the vast majority of their patients. One GP in Site 6 referred to cancer genetics provision as "a bit of a Cinderella-type service: if it was available you might use it, but if it wasn't then as a GP you weren't too concerned." This resulted in severe difficulties for pilot staff in accessing general practice-based professionals to provide information and education on the new services they were setting up. Practice managers acted effectively as 'gatekeepers' to GPs; GPs themselves were in some cases fiercely protective of their practice nurses' time:

"Some practices have regular meetings and all members of the team work closely together and everyone is involved, and then you will get another practice down the road who never have a practice meeting. [...] Practice nurses are probably the ideal person [to identify and refer patients to the pilot] but they are employed by their practice and some of the GP practices are very 'No, my practice nurse does this, this, and this if they are on my must-do list, she hasn't got time to spend three hours with you on education', and that's really difficult." (Pilot nurse, Site 6)

Some respondents attributed these differences to age of GPs, arguing that the more recently graduated would have greater understanding of the relevance of genetics to their day-to-day practice; for others, it seemed more a matter of differences of organisational culture between practices.

Pilot staff developed various strategies for "getting into the GP world," as a nurse in Site 2 put it. Pilots that counted GPs among their staff found themselves at an advantage in this regard, 
building on existing relationships with other local GPs. Conversely, respondents from nurse-led pilots sensed that some GPs were less willing to refer to their services, and indeed a surgeon in Site 7 noted that certain GPs continued to refer patients with suspected family histories of cancer directly to him rather than through the unitary care pathway instituted by the nurse-led pilot and supported by the PCT. However, over time, pilots did generally find ways of building trust among GPs, who were important not only as referrers, but also for their influence on future commissioning decisions. Well known practitioners within the pilots, or supporters within the PCT, sometimes brought the necessary social capital to encourage GPs and others to engage. Persistence often eventually resulted in access to GP practices, though GPs' engagement often came at a cost- "money or a free lunch," as a pilot nurse in Site 4 wryly commented-reflecting the lack of intrinsic interest in genetic matters (the cause of which is considered in the next section). Service-development projects based in secondary care found that extensive publicity and persistent contact with GPs was the only way to improve knowledge of provision that had been previously somewhat remote from day-to-day primary-care practice, and commissioned at a regional specialised level, rather than locally.

The reluctance of primary care practitioners to engage with genetics was not just attributable to lack of interest. Centrally mandated priorities and changes inevitably commanded PCTs' attentions, and genetics seemed a comparatively low priority. GPSIs faced a succession of different managers (four in the case of Site 9's GPSI) as PCTs contended with the demands of central government and the reconfiguration of their boundaries. Meanwhile, the introduction of the new GP contract concentrated GPs' minds on areas covered by the Quality and Outcomes Framework (QOF), which did not include genetics.

In some cases, it was possible to emphasise alignments between pilots' work and centrally determined priorities, playing up points of confluence which PCTs were otherwise happy to ignore:

"I don't think [genetics] is that decoupled from things like the NSF [National Service Framework]; I just think that people haven't really picked up on that. [...] PCTs have not particularly picked up on them because until [laughs] they're forced to take notice, they've probably got enough things on their plate." (Clinical geneticist, Site 3)

The solution, then, was "to be more creative in how we sell it to the PCTs," as a nurse in the same pilot put it. Such alignments were not so evident for all projects, however, and some had to rely on the agency of patients themselves to drive primary care practitioners to engage with genetic issues and make referrals to the new services. The clinical geneticist in Site 1 observed that:

"patients with [this condition] certainly are far more informed and they are definitely tripping up far more frequently to their own doctors and saying, 'I want to be referred'. [...] I love pushy patients. They don't bother me, because all of our patients are pushy: that's why they get to see us."

The onset of the condition in question, though, tended to take place in early to mid-adulthood, and respondents elsewhere noted that gaining public engagement was not always so straightforward, especially in socio-economically deprived areas.

\section{The place of genetics within primary care practice}

For the kinds of reasons noted above, many hospital-based practitioners felt that provision needed to be primary care-led. As a genetic counsellor in Site 6 put it, "if we go out to people, they just switch off: they're not interested in genetics, they have a lot else going on. [...] It has to come from primary care." However, a number of connected features of primary care practice militated against efforts to foster this kind of involvement.

For a start, most GPs and other primary care practitioners had limited contact with genetics services. As several GPs pointed out, most direct referrals to clinical genetics departments would be for children with unknown conditions, where genetics was seen to have a 
clear role in diagnosis, and in informing parents' pre-conceptual decision-making. Most adults with conditions that might have a genetic component would be referred to 'mainstream' clinical specialists in secondary care, and so direct contact between primary care and clinical genetics involving these cases was limited. Consequently, in relation to non-paediatric cases, in particular, detailed knowledge of genetics was not seen as directly relevant to primary care practice for the large majority of patients:

"You can only hold so much knowledge in your brain, and you have to prioritise the knowledge which (a) you are likely to come across or (b) where if you come across something very rare, the consequences are very great. [...] [Those who] think the GP should know more about genetics [...] don't understand the breadth of the workload, the topics with which we work." (GP, Site 2)

Lack of knowledge about genetics and lack of perceived relevance to day-to-day practice reinforced each other. ${ }^{9,13}$ Consequently, for many primary care practitioners, the most appropriate solution, befitting the perceived level of need for extra genetics knowledge in primary care, was a rational division of labour that would see the development of specialist individuals or teams within primary care, which other primary care practitioners could draw on as necessary without developing genetics knowledge themselves.

This, indeed, was the model pursued by many of the funded projects, which involved primary care-based teams (or individual GPSIs) providing an intermediary service that bridged the gap with clinical genetics specialists - though not always in a way that those specialists thought appropriate. ${ }^{14}$ Other projects, though, attempted to instil a consciousness of the more wide-ranging relevance of genetic knowledge among primary care practitioners. One GPSI, for example (Site 8), held workshops to demonstrate the relevance of genetics to everyday primary care practice, and to assist others to "think genetic" and keep ahead of the 'genomic revolution'. This approach to the mainstreaming of genetics within primary care, in line with the ethos of the White Paper, was endorsed by the $\mathrm{DH}$, which saw the pilot programme as a means of raising the profile of genetics throughout the Health Service. In particular, this meant demonstrating its centrality to general practice, for example, in the management of many chronic diseases, alongside non-genetic environmental factors:

“The common chronic diseases, most genetics services don't want to go there. [...] They're concentrating on learning about more and more rare conditions, and they're not really interested in getting into the multi-factorial stuff. We don't know whether that's going to deliver, but we need to have a workforce in primary care that's ready to take it on if it does, or able to critique it and say, 'We won't use it'—but they've got to know enough about it." (DH policy lead)

Crucially, then, this vision implied a much more proactive engagement with genetic knowledge, as it related to the aetiology of common chronic conditions, on the part of all primary care practitioners. It was not about the creation of small groups of primary care-based specialists to whom GPs and others could turn on those comparatively rare occasions when clinical genetics affected specific patients.

However, most projects were framed in ways that mirrored the existing set-up, and thus consolidated the prevailing institutional arrangements that saw clinical genetics as either an esoteric speciality relevant to a small number of patients, or as something accessed via other clinical areas such as cancer. Projects provided more accessible care pathways for patients falling into these categories that were often appreciated by GPs, but they did not reframe genetics as something that was of more direct, wide-ranging interest in routine primary care practice. Those projects that did attempt to achieve this found themselves unable to challenge the lack of interest that saw genetics as of peripheral concern to primary care. The GPSI in Site 8, for example, concluded that despite his best efforts to promulgate the importance of genetics to primary care, for most practitioners, it remained "a speciality for people with rare diseases." 
The framing of most projects in terms of specific areas of clinical genetic practice, meanwhile, was often more effective in obtaining buy-in from GPs and other primary care practitioners, but, in doing so, consolidated the perception of genetics as something specialised and of narrow relevance. In Site 4, for example, a new cancer genetics referral system was introduced which, as a GP enthused, "allows people in primary care to put patients into roughly three pigeon holes," and avoid making inappropriate referrals to oncologists or clinical genetics specialists. The system had been embraced by primary care practitioners, the GP argued, because it meant that "we can stand behind the genetics service and their referral guidelines [...] given that genetics is a relatively new discipline to a lot of GPs who probably wouldn't have the confidence to vet people out." A GP in Site 7 agreed that this kind of approach was appropriate, since

"there's not a great deal of skill out there anyway. [...] The majority of GPs are going to be a lot older than me, so their taught knowledge of genetics is going to be potentially poorer. So 'No', I don't think there are any skills that this will undermine-there is not much skill there to be deskilled anyway."

Ironically, then, engagement with primary care was most successful when it did not challenge the perception of genetics as specialist and peripheral-something for others to concern themselves with-but sustained it. The implications of this are taken up in the Discussion.

\section{Sustainability, commissioning and long-term prospects}

Even projects that were successful in 'mainstreaming genetics', in their own terms, faced problems in securing ongoing funding. At the time that many staff were looking to sustain pilots' work, the NHS was undergoing significant upheaval, involving PCT reorganisations and the insistence from central government that NHS organisations rein in deficits. In this climate, it was difficult to obtain funding for new services, even if — in the long term — they might offer savings through earlier detection and better management of inherited illnesses. Some pilots managed to deal with this challenge. Site 4, for example, managed to demonstrate a shorter-term saving through more appropriate use of screening services, while in Site 1 provision was integrated into the business plan of the mainstream disease area of the hosting hospital.

However, the challenges around ongoing commissioning arrangements were not limited to short-term turmoil caused by NHS reorganisation. They were also associated with the structures of the NHS, notably the split between mainstream commissioning, as carried out by individual PCTs, and specialist commissioning, performed regionally by PCT consortia. Most genetics services were traditionally specialist-commissioned, but as pilots explicitly aimed at bridging the gap with mainstream provision, the situation was ambiguous for these new services. Knowledge of genetics issues among mainstream commissioners remained, according to specialist commissioners, rather deficient, despite their increasing relevance to wider health care provision, and this did not bode well for 'world-class commissioning' 15 in this field:

"I think [PCT-level commissioners] will feel fairly shortly that they're losing control. [...] If genetics goes the way it appears to be heading, there will be a lot more pressure on commissioners to [improve their knowledge]. If they don't, they will have lost control and will not understand it and will be at the mercy of these scientists who speak a totally different language." (Specialist commissioner, Site 4)

Yet service providers, too, had fears about this brave new commissioning world, and especially the prospect of integration with other specialities, losing the protection afforded by specialist commissioning arrangements:

"When genetics just does its own bids to a pot of money for specialised services it's quite neat and tidy and specific. If we become more mainstreamed and we're just a sub-bid of a cardiology bid or an obesity-clinic bid or whatever, then we might well end up getting nothing, partly because we get lost against the headline person making the bid, and genetics is just a line at the bottom. If the full amount of funding isn't allocated then we'll probably 
be one of the things that gets chopped off." (General manager in clinical genetics department, Site 4)

Practice-based commissioning, in which purchasing decisions are devolved from PCTs to groups of GPs, also seemed a mixed blessing for pilot services. Respondents from hospitalbased pilots expressed concern that practice-based commissioners might be too occupied with higher profile and costlier issues to devote attention to the benefits of their services, and their potential incremental improvements in patient experience and reductions in cost. However, there was a sense from PCT-based pilots that practice-based commissioners would be happy to fund small-scale community-based services, if given sufficient backing from PCT management, since they fitted well with the wider commissioning agenda relating to providing care 'closer to home'. Practice-based commissioning, then, neither seemed to threaten these service developments, but nor did it generally provide the drive needed to become an established part of local service provision.

There was particular concern about the prospects for 'mainstreaming' genetic knowledge and provision in an NHS which remained, for many, dominated by shorter-term issues resulting in the neglect of preventive measures. A cancer network manager in Site 5 summed up the situation in relation to cancer, a field perhaps more subject than most to a forceful centrally driven performance management regime:

"Genetics is a buzzword: everyone says, 'Prevention is better than cure', understanding that cancer networks focus far too much of their attention on treatment. So, in some ways, the vibes are quite good: ideal to have these pilots looking at how you can reach people effectively. [But] if I go to the PCTs with any service development, unless it is linked to a target or is completely unavoidable, they just say, 'You must be mad'. What they saywhich is true-is: 'We haven't got the money to run the services we've got: why would we fund new services?"”

PCTs, despite their public health focus, found it difficult to raise the profile of genetics given more pressing concerns around acute care commissioning and chronic disease management. This was compounded for some by departmental divisions within PCTs, which made it difficult for pilots to appeal:

"I see this as a public health target, but I sit in a different department doing commissioning and development, and you've got the team of people delivering the service within the clinical service department. [...] People working in the NHS are just surviving managing disease, and if these projects can raise the profile, we've achieved something, but I want more than that. The whole thing has to shift to focus onto prevention, not disease management." (Pilot manager, Site 7)

\section{Discussion}

The findings presented above present a necessarily sweeping overview of the issues facing efforts to bring genetics into primary care. Other publications from the evaluation offer a more sustained analysis of some of these issues. ${ }^{14,16,17,18,19}$ Nevertheless, some important messages are evident.

First, the findings mirror those of various studies highlighting the difficulties that arise in efforts to promote joined up working between agencies and sectors, especially the impact of vertical governance arrangements on the willingness of different stakeholders to engage. ${ }^{20,21} \mathrm{~A}$ notable difference, though, between the genetics pilots and, for example, efforts to join up health and social care services to improve discharge arrangements for older people, 5,22 was the general lack of engagement from significant numbers of primary care practitioners. Whereas, for all their disputes over responsibility, discharge arrangements are regarded as a matter of mutual concern for the acute, primary and social care sectors, here the prevalent view seemed to be that genetics was not of particular relevance to primary care, despite acknowledgement of the potential 
implications of the knowledge arising from the 'new genetics'. The efforts of Site 8's GPSI, for example, to instil a consciousness of the relevance of genetics to everyday primary care practice succeeded, by his own admission, in engaging only a few practitioners. An absence of recognition of responsibility would seem to be a key barrier to top-down efforts of this kind to encourage knowledge sharing.

The existing organisation of genetics in the Health Service partly explains this absence. Direct contact between primary care and clinical genetics departments was rare, except for a small group of patients; in other cases, specialists in secondary care would usually mediate the relationship. Since many of the pilots, including those based in primary care, focused on specific areas of clinical genetics and provided simplified care pathways that minimised the level of knowledge required by GPs and other referrers, they actually consolidated the view of genetics as of peripheral concern to primary care, even as they secured the buy-in they required from primary care practitioners. Those projects that sought to improve knowledge by explaining the rising relevance of genetics to day-to-day primary care practice found that, given the institutionalised marginality of genetics from primary care, most practitioners had more pressing priorities. Paradoxically, then, it seems that if the aim is to achieve something of a 'cultural change' in the primary care workforce, "building expertise and capacity specifically in primary care" to deal with the anticipated 'genomic revolution', through a programme of genetics pilots is an ineffective way of achieving change. If the desired outcome of policymakers is - as the White Paper suggests - primary care that sees genetics as central to its everyday practice, then piloting new clinical genetics services, even if based in primary care, seems counter-productive, serving only to confirm the marginal status of genetics. Rather, this kind of transformatory change would seem to need to be incorporated into wider policy efforts at reconfiguring the role of general practice and creating a primary care-led NHS, so that those pilots seeking to address the general level of knowledge among primary care practitioners are helped, rather than hindered, by wider changes.

These changes include the consequences of recent UK policy developments such as the new NHS GP contract, which focused GPs more closely on work rewarded by the QOF element in the contract, and also seemed to impair pilots' ability to access other general practice-based staff-though there were differences between general practices in this regard, apparently reflecting organisational culture. ${ }^{23}$ This reflects findings elsewhere in relation to the consequences for surveillance, particularly of lower-status staff such as nurses and health care assistants, of the new GP contract, involving more intrusive intra-practice management regimes. ${ }^{24}$ While access to general practice staff was problematic for some pilots, simple guidance for dealing with patients with suspected inherited conditions was largely appreciated by GPs- "a care pathway and a referral guideline, that's it," as the clinical geneticist in Site 4 summarised. Once again, though, the result was that genetics remained marginal to the consciousness of most primary care practitioners, even if the introduction of protocols had the potential to improve patient care.

Meanwhile, the reorganisation of the NHS and associated managerial upheavals meant that genetics was at best a side issue for most PCT managers, too. Though, on the one hand, this might be seen as a consequence of unfortunate timing, it should also be acknowledged that such problems are likely to be repeated given the seemingly perpetual state of reorganisation of the NHS - in Walshe's memorable phrase, "an organizational shantytown in which structures and systems are cobbled together or thrown up hastily in the knowledge that they will be torn down again in due course." ${ }^{25}$ All in all, a panoply of policy interventions-centrally driven performance management, marketisation, and continuous structural revolution-seemed to work against the joining up and knowledge-sharing drive of the White Paper, mirroring the policy barriers to the move towards public-service 'modernisation' more widely, ${ }^{4,16}$ whereby "different policy strands have created unhelpful dissonances." 26 
Particularly notable in the context of genetics was the dissonance between mainstream (PCT-based) and specialist (PCT consortium-based) commissioning. The efforts to enfranchise individual PCTs in the development of genetics services exposed the knowledge deficit of their commissioners, who had never previously needed to engage with the area. This left both pilots and existing specialist services uneasy about the changing commissioning scene, with concern among clinical genetics departments about the possibility of resources moving away from tertiary care once block funding was replaced by per case commissioning arrangements. For PCTs, meanwhile, their relatively small size left them unable to plan their genetics provision strategically, and for all the rhetoric about a primary care-led NHS, the power of hospital-based providers remained great, given their sway over knowledge and historically entrenched positional power. Concerns over the capacity and knowledge of mainstream commissioners in this field, and the loss of what Paton calls "meso-level planning" ${ }^{26}$ resulting from the division of commissioning responsibilities, threatened a lack of strategic direction in service provision, and suggested that in genetics, at least, the challenges identified for 'world-class commissioning' by Ham are indeed profound. ${ }^{15}$

\section{Conclusion}

The pilots funded through this programme had varying degrees of success in establishing and sustaining their work to raise awareness and embed genetics within primary care practice, but the challenges faced were common to many of them. Policies that mitigate competing pressures for practitioners might assist the uptake of initiatives of this kind. A range of strategies deployed by pilot staff such as extensive and persistent educational work, alignment with national targets and drawing on the agency of interested patients or influential local stakeholders resulted in some local successes in achieving change. Such strategies, however, were not viable for all pilots, and persistent features of NHS policy, organisation and culture meant that if initiatives such as these were to realise the ambitions of the White Paper to infuse genetics knowledge and provision throughout the Health Service, they needed considerably more support from policy makers and local managers alike, and arguably needed to be framed not in terms of clinical genetics provision seen as marginal by many primary-care practitioners, but as a key part of more general reforms of the role of primary care in the NHS.

\section{Acknowledgements}

The evaluation from which this paper is drawn was funded by the UK Department of Health. We would like to thank the research participants for their time and input, and two anonymous referees for their helpful comments

\section{References}

${ }^{1}$ Secretary of State for Health. Our Inheritance, Our Future: Realising the Potential of Genetics in the NHS. London: The Stationery Office, 2003.

${ }^{2}$ Ferlie E, Pettigrew A. Managing through networks: some issues and implications for the NHS. British Journal of Management 1996;7:S81-99.

${ }^{3}$ Bate P. Changing the culture of a hospital: from hierarchy to networked community. Public Adm 2000;78:485-512.

${ }^{4}$ Currie G, Suhomlinova O. The impact of institutional forces upon knowledge sharing in the UK NHS: the triumph of professional power and the inconsistency of policy. Public Adm 2006;84:1-30.

${ }^{5}$ Regen E, Martin G, Glasby J, Hewitt G, Nancarrow S, Parker H. Challenges, benefits and weaknesses of intermediate care: results from five UK case study sites. Health Soc Care Community 2008;16:629-37. 
This is the final draft, after peer-review, of a manuscript published in Journal of Health Services

Research \& Policy. The definitive version, detailed above, is available online at www.rsmjournals.com. (C) 2009 Royal Society of Medicine Press Limited

${ }^{6}$ Hudson B. Sea change or quick fix? Policy on long-term conditions in England. Health Soc Care Community 2005;13:378-85.

${ }^{7}$ Emery J, Hayflick S. The challenge of integrating genetic medicine into primary care. BMJ 2001;322:1027-30.

${ }^{8}$ Qureshi N, Modell B, Modell M. Raising the profile of genetics in primary care. Nat Rev Genet 2004;5:783-90.

${ }^{9}$ Robins R, Metcalfe S. Integrating genetics as practices of primary care. Soc Sci Med 2004;59:22333.

${ }^{10}$ Hadfield SG, Horara S, Bhatnagar D et al. Significant factors in implementing cascade testing for familial hypercholesterolaemia. Atherosclerosis 2007;194:279-80.

${ }^{11}$ Eeles R, Purland G, Maher J, Evans DG. Delivering cancer genetics services - new ways of working. Fam Cancer 2007;6:163-7.

${ }^{12}$ Eisenhardt KM. Building theory from case study research. Acad Manage Rev 1989;14:532-50.

13 Kumar S, Gantley M. Tensions between policy makers and general practitioners in implementing new genetics: grounded theory interview study. BMJ 1999;319:1410-13.

${ }^{14}$ Martin GP, Currie G, Finn R. Reconfiguring or reproducing intra-professional boundaries? Specialist expertise, generalist knowledge and the 'modernization' of the medical workforce. Soc Sci Med 2009;68:1191-8.

15 Ham C. World class commissioning: a health policy chimera? J Health Serv Res Policy 2008;13:116-21.

${ }^{16}$ Currie G, Finn R, Martin G. Accounting for the 'dark side' of new organizational forms: the case of healthcare professionals. Hum Relat 2008;61:539-64.

${ }^{17}$ Currie G, Martin G, Finn R. Professional competition and modernising the clinical workforce in the NHS: possibilities and limits to the development of the specialist generalist in primary care. Work, Employment \& Society in press.

${ }^{18}$ Martin GP, Currie G, Finn R. Leadership, service reform, and public-service networks: the case of cancer-genetics pilots in the English NHS. Journal of Public Administration Research \& Theory in press.

${ }^{19}$ Martin GP. Representativeness, legitimacy and power in public involvement in health-care management. Soc Sci Med 2008;67: 1757-65.

${ }^{20}$ Rummery K, Coleman A. Primary health and social care services in the UK: progress towards partnership? Soc Sci Med 2002;56:1773-82.

${ }^{21}$ Glendinning C. Breaking down barriers: integrating health and care services for older people in England. Health Policy 2003;65:139-151.

${ }^{22}$ Glasby J, Martin G, Regen E. Older people and the relationship between hospital services and intermediate care: results from a national evaluation. J Interprof Care 2008;26:639-49.

${ }^{23}$ Checkland K. Understanding general practice: a conceptual framework developed from case studies in the UK NHS. BrJ Gen Pract 2007;57:56-63.

${ }^{24}$ McDonald R, Harrison S, Checkland K. Incentives and control in primary health care: findings from English pay-for-performance case studies. J Health Organ Manag 2008;22:48-62.

${ }^{25}$ Walshe K. Foundation hospitals: a new direction for NHS reform? J R Soc Med 2003;96:106-10.

${ }^{26}$ Paton C. New Labour's State of Health: Political Economy, Public Policy and the NHS. Aldershot: Ashgate, 2006. 